\title{
ARTICLE \\ An inverse relationship between cortical plasticity and cognitive inhibition in late-life depression
}

Jennifer I. Lissemore ${ }^{1,2}$, Hayley R. C. Shanks (iD) ${ }^{1}$, Meryl A. Butters ${ }^{3}$, Apoorva Bhandari ${ }^{1}$, Reza Zomorrodi ${ }^{1}$, Tarek K. Rajji ${ }^{1,2,4}$, Jordan F. Karp ${ }^{3,5}$, Charles F. ReynoldsIII ${ }^{3}$, Eric J. Lenze ${ }^{6}$, Zafiris J. Daskalakis ${ }^{1,2,4}$, Benoit H. Mulsant ${ }^{2,4}$ and Daniel M. Blumberger $^{1,2,4}$

Executive dysfunction is a common and disabling component of late-life depression (LLD), yet its neural mechanisms remain unclear. In particular, it is not yet known how executive functioning in LLD relates to measures of cortical physiology that may change with age and illness, namely cortical inhibition/excitation and plasticity. Here, we used transcranial magnetic stimulation (TMS) to measure cortical inhibition/excitation $(n=51)$, and the potentiation of cortical activity following paired associative stimulation, which is thought to reflect long-term potentiation (LTP)-like cortical plasticity $(n=32)$. We assessed the correlation between these measures of cortical physiology and two measures of executive functioning: cognitive inhibition, assessed using the Delis-Kaplan Executive Function System Color-Word Interference ["Stroop"] Test, and cognitive flexibility, assessed using the Trail Making Test. Correlations with recall memory and processing speed were also performed to assess the specificity of any associations to executive functioning. A significant correlation was found between greater LTP-like cortical plasticity and poorer cognitive inhibition, a core executive function $\left(r_{p}=-0.56, p<0.001\right)$. We did not observe significant associations between cortical inhibition/excitation and executive functioning, or between any neurophysiological measure and cognitive flexibility, memory, or processing speed. Our finding that elevated cortical plasticity is associated with diminished cognitive inhibition emphasizes the importance of balanced synaptic strengthening to healthy cognition. More specifically, our findings suggest that hyper-excitability of cortical circuits following repeated cortical activation may promote inappropriate prepotent responses in LLD. LTP-like cortical plasticity might therefore represent a neural mechanism underlying an inhibitory control cognitive endophenotype of LLD.

Neuropsychopharmacology (2019) 44:1659-1666; https://doi.org/10.1038/s41386-019-0413-9

\section{INTRODUCTION}

Late-life depression (LLD) is an understudied yet debilitating disorder in older adults (i.e., $>60$ years of age). One cognitive symptom consistently associated with LLD is executive dysfunction. Executive functioning encompasses top-down cognitive control, including the inhibition of prepotent responses, and the flexible switching between tasks (for a review of core executive functions, see [1]). Several studies have shown impaired executive functioning in LLD patients compared to similarly aged healthy controls [2-6]. More specifically, impairments in inhibitory control $[7,8]$ and cognitive flexibility [9] have been linked to LLD, although inconsistent findings [10] suggest heterogeneous cognitive profiles amongst patients. Impairment within these executive domains is associated with a worse prognosis [11]; for example, inhibitory control deficits are associated with increased risk of suicide in LLD patients [12]. Moreover, executive dysfunction often persists following antidepressant treatment [13]. An understanding of the biological mechanisms related to executive functioning will therefore be important for the development of treatments that address this key LLD symptom.

The neurobiological basis of executive functioning in LLD is not fully understood. Previous studies have associated executive dysfunction with structural pathologies such as white matter hyperintensities [2] and reduced white matter integrity in frontal tracts [14]. Very few studies have examined the relationship between functional brain processes and executive functions in LLD. One functional magnetic resonance imaging study of LLD patients found a link between executive dysfunction and abnormal inter-network functional connectivity, which was specific to executive functioning and not recall memory [15]. A few electroencephalography (EEG) studies of LLD have also linked executive functioning to the abnormal latency or amplitude of cortical event-related potential components $[3,7,16]$. Although LLD involves the interplay between depression and healthy aging, studies of LLD have yet to explore the relationship between executive functioning and cortical physiological processes that have been implicated in both depression and aging.

Cortical physiological processes that have been associated with both the pathophysiology of depression and advancing age include glutamate receptor-mediated cortical plasticity and $\gamma$-aminobutyric acid (GABA) receptor-mediated cortical inhibition. In a healthy brain, synaptic plasticity is regulated by a host of mechanisms to avoid the induction of excessively

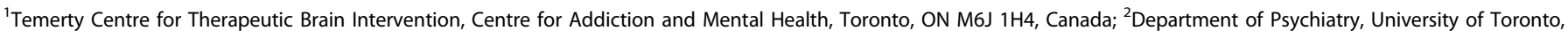
Toronto, ON M5T 1R8, Canada; ${ }^{3}$ Department of Psychiatry, University of Pittsburgh School of Medicine, Pittsburgh, PA, USA; ${ }^{4}$ Campbell Family Mental Health Research Institute,

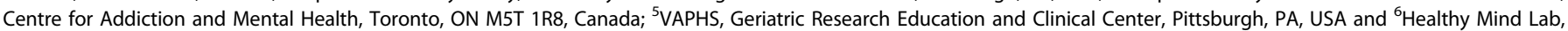
Department of Psychiatry, Washington University School of Medicine, St Louis, MO, USA Correspondence: Daniel M. Blumberger (daniel.blumberger@camh.ca)
}

Received: 31 December 2018 Revised: 27 April 2019 Accepted: 1 May 2019

Published online: 9 May 2019 


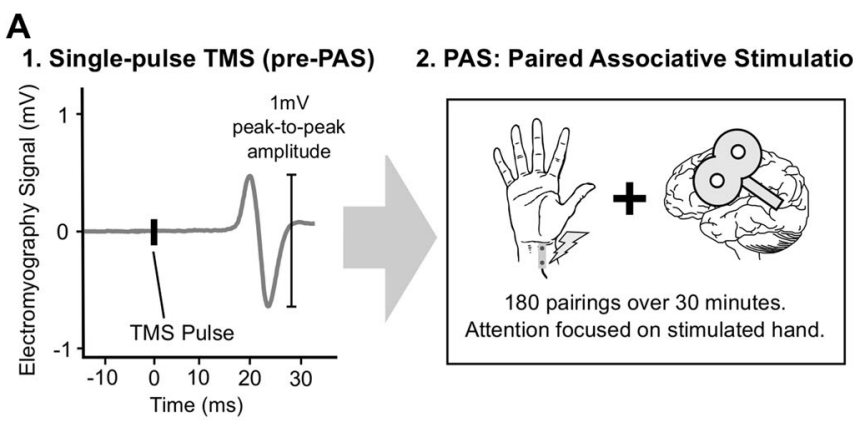

\section{Single-pulse TMS (post-PAS)}

Short-Interval Cortical Inhibition

ICF:

Intracortical Facilitation

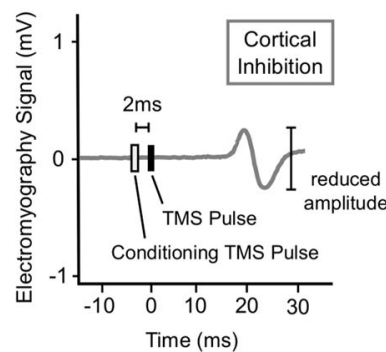

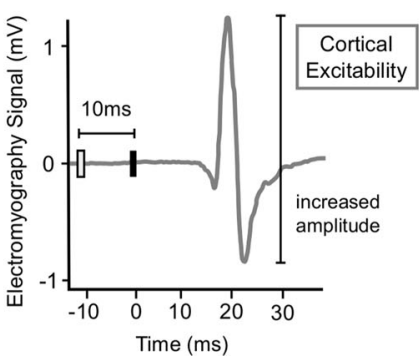

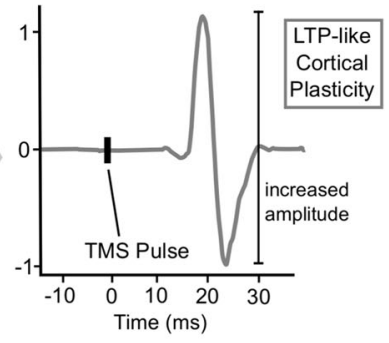

C

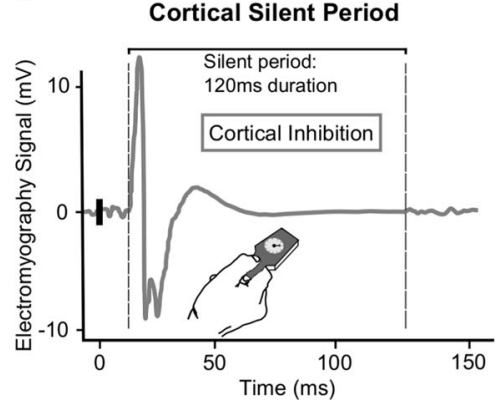

Fig. 1 Illustration of the transcranial magnetic stimulation (TMS) paradigms. a Paired associative stimulation (PAS): peripheral nerve stimulation is repeatedly paired with TMS, which amplifies motor evoked potential (MEP) amplitudes for $\sim 1 \mathrm{~h}$. PAS reflects long-term potentiation-like cortical plasticity. b Short-interval cortical inhibition (SICI) and intracortical facilitation (ICF): a subthresold TMS pulse precedes a suprathreshold TMS pulse, which dampens or enhances the MEP amplitude, respectively. SICI probes inhibitory cortical circuits, and ICF excitatory cortical circuits. c Cortical silent period: a TMS pulse during muscle contraction results in a period of silenced electromyography signal, which reflects inhibitory cortical circuits

high or low firing rates [17], and inhibitory and excitatory neurotransmission are balanced through feedforward and feedback mechanisms [18]. Cortical plasticity and inhibition have been found to be dysregulated in younger adults with depression [19, 20], and in older adults without depression $[21,22]$. However, at the intersection of aging and depression, LLD presents with a heterogeneous symptom profile, and the involvement of cortical plasticity and inhibition/excitation in LLD is less clear $[23,24]$. Specific features of LLD, such as cognitive endophenotypes, may more closely relate to abnormalities in cortical physiology than a complex diagnosis of LLD [25]. To narrow down the problem, we need to assess how cortical plasticity and inhibition/excitation relate to cognitive endophenotypes within LLD that have primarily cortical origins, such as executive functioning [26, 27].

One powerful technique for measuring cortical physiological processes in humans is transcranial magnetic stimulation (TMS). Using TMS, we can non-invasively assess cortical plasticity in vivo in LLD patients (Fig. 1a). In a paired associative stimulation (PAS) paradigm, single pulses of TMS are repeatedly paired with somatosensory afferent stimulation, both of which activate postsynaptic pyramidal cells in the motor cortex. Stimulation is timed such that the TMS and somatosensory signals arrive synchronously at the cortex. This form of temporally and spatially linked repeated stimulation mimics spike-time-dependent plasticity paradigms [28]. We can use PAS to measure long-term potentiation (LTP)-like cortical plasticity by measuring the potentiation of cortical excitability (motor evoked potential [MEP] amplitude) after PAS. Like LTP, PAS-induced potentiation of cortical excitability can be induced rapidly ( $30 \mathrm{~min}$ ), lasts $>1 \mathrm{~h}$, and depends on the activation of $\mathrm{N}$-methyl-D-aspartate (NMDA) receptors $[29,30]$. PAS-induced potentiation putatively reflects the sustained, yet reversible, strengthening of many synapses within a cortical area that results from the repeated activation of that cortical area.
TMS paradigms have also been developed to measure cortical inhibition and excitation. In a short-interval cortical inhibition (SICl) paradigm (Fig. 1b), paired subthreshold and suprathreshold TMS pulses are timed precisely to suppress cortical excitability, reflecting $\mathrm{GABA}_{\mathrm{A}}$ receptor-mediated inhibitory neurotransmission [31, 32]. Conversely, in an intracortical facilitation (ICF) paradigm (Fig. 1b), the paired TMS pulses are timed to enhance cortical excitability, reflecting NMDA receptor-mediated excitatory neurotransmission [31, 32]. GABA $A_{B}$ receptor-mediated cortical inhibition can also be probed using a cortical silent period paradigm (Fig. 1c) $[32,33]$, in which a single TMS pulse is delivered during muscle contraction, so as to produce a measurable "silent period" in the electromyography signal. Lastly, the resting motor threshold, defined as the minimum stimulation intensity that elicits an MEP of a particular size, can be used to assess cortical excitability. Although the above TMS paradigms have been used to investigate a range of neuropsychopathologies [34], TMS has not yet been applied to study the cortical mechanisms underpinning LLD endophenotypes.

Here, we used TMS to measure LTP-like cortical plasticity, cortical inhibition, and cortical excitation in LLD patients, and we explored the relationship between these TMS measures and executive functioning. We hypothesized that cortical plasticity and inhibition/excitation would be associated with two core executive functions in LLD: cognitive flexibility and cognitive inhibition. To establish the specificity of any relationships between cortical physiology and executive functioning, we also examined the relationship between the above TMS measures and episodic memory and processing speed.

\section{MATERIALS AND METHODS}

Participants

Patients were recruited from the National Institute of Mental Health (NIMH)-sponsored IRL-GRey clinical trial of aripiprazole 
augmentation pharmacotherapy at the Centre for Addiction and Mental Health (CAMH), Toronto. Patients completed all TMS and neurocognitive assessment at baseline, before initiating a pre-trial with open-label venlafaxine (ClinicalTrials.gov identifier: NCT00892047) [35, 36]. All patients were aged 60 years and older, had a diagnosis of major depressive disorder as per the Structured Clinical Interview for DSM-IV (Diagnostic and Statistical Manual of Mental Disorders, 4th Edition) [37], and scored $\geq 15$ on the Montgomery-Asberg Depression Rating Scale (MADRS) [38]. Exclusion criteria included a comorbid psychiatric diagnosis other than an anxiety disorder, an unstable medical illness, anticonvulsant use, or dementia. Clinical evidence of dementia included a Mini Mental State Examination [39] score <21, as well as the assessment of a geriatric psychiatrist, medical records, and an informant's interview in unclear cases. Patients were excluded if taking a psychotropic medication that could not be safely tapered; exceptions included lorazepam $\leq 2 \mathrm{mg} /$ day (or another benzodiazepine at an equivalent dosage), zopiclone $\leq 7.5 \mathrm{mg} / \mathrm{day}$, or trazodone $\leq 50 \mathrm{mg} /$ day. Due to tapering prior to treatment initiation, some patients were still taking a low dose of antidepressant at the time of testing. Treatment resistance was assessed using the Antidepressant Treatment History Form [40], and patients with any level of treatment resistance were included in this study. Medical illness burden was assessed using the Cumulative Illness Rating Scale for Geriatrics [41], and general anxiety symptoms using the Brief Symptom Inventory (anxiety subscale) [42]. Patient demographic, clinical, and neurocognitive data listed in Table 1 were obtained.

All study procedures were approved by the CAMH Research Ethics Board in accordance with the Declaration of Helsinki, and all participants provided written, informed consent.

TMS and electromyography measures

The TMS measures reported here adhere to a TMS methodological checklist established by international experts [43]. Surface electromyography was recorded continuously using disposable $9 \mathrm{~mm}$ electrodes positioned at the abductor pollicus brevis muscle (active electrode) and the interphalangeal joint of the thumb (reference electrode). A ground electrode was placed on the upper forearm. A figure-of-eight TMS coil (70 $\mathrm{mm}$ loop diameter) was positioned over the left motor cortex "hot spot" to evoke maximum MEP strength. The TMS coil was held tangentially to the head, with the handle angled $\sim 45^{\circ}$ from the midline to induce a posterior-anterior current, and the coil position was marked on the scalp. Monophasic pulses were delivered using two Magstim 200 stimulators (Magstim, Whitland, UK) connected by a Bistim module.

Patients were instructed to remain relaxed with their eyes open. Electromyography recordings were monitored to ensure relaxation of the abductor pollicus brevis muscle. Resting motor threshold was first established as the minimum TMS intensity that elicits $a \geq 50 \mu \mathrm{V}$ MEP in $5 / 10$ trials. The TMS intensity that elicited on average a $\sim 1 \mathrm{mV}$ peak-to-peak MEP amplitude was then established as the intensity for the suprathreshold TMS test pulse in SICI, ICF, and PAS paradigms (described below, see Fig. 1).

In the $\mathrm{SICl}$ and ICF paradigms, a subthreshold conditioning pulse ( $80 \%$ resting motor threshold) preceded the suprathreshold test pulse with an interstimulus interval of $2 \mathrm{~ms}(\mathrm{SICl})$ or $10 \mathrm{~ms}$ (ICF). Participants underwent 36 trials; 12 for each condition in a pseudorandom order (test pulse alone, and test pulse either 2 or $10 \mathrm{~ms}$ after the conditioning pulse). Peak-to-peak MEP amplitudes were calculated for each trial and averaged for each condition. The $\mathrm{SICl}$ and ICF ratios were calculated as the average conditioned MEP amplitude ( 2 or $10 \mathrm{~ms}$ between TMS pulses)/the average unconditioned MEP amplitude (test pulse alone).

In the cortical silent period paradigm, a suprathreshold test pulse ( $140 \%$ of resting motor threshold) was administered over 10 trials during tonic muscle contraction $(20 \%$ of maximum
Table 1. Demographic, clinical, and neurocognitive information for all late-life depression patients, and for those patients who underwent PAS

\begin{tabular}{|c|c|c|}
\hline Demographic and clinical information & $\begin{array}{l}\text { Full sample } \\
(n=51)\end{array}$ & $\begin{array}{l}\text { PAS subset } \\
(n=32)\end{array}$ \\
\hline Age (years) [range] & $\begin{array}{l}66.6 \pm 5.9 \\
{[60-89]}\end{array}$ & $\begin{array}{l}67.2 \pm 5.2 \\
{[60-78]}\end{array}$ \\
\hline Sex & $30 \mathrm{~F} / 21 \mathrm{M}$ & $17 \mathrm{~F} / 15 \mathrm{M}$ \\
\hline$\%$ Caucasian & $92.2(n=47)$ & $90.6(n=29)$ \\
\hline Education (years) & $13.8 \pm 2.7$ & $14.1 \pm 2.7$ \\
\hline Handedness & $47 \mathrm{R} / 4 \mathrm{~L}$ & $29 \mathrm{R} / 3 \mathrm{~L}$ \\
\hline MADRS score & $25.3 \pm 5.6$ & $24.7 \pm 5.1$ \\
\hline $\begin{array}{l}\text { Duration of current depressive } \\
\text { episode (weeks) [range] }\end{array}$ & $\begin{array}{l}87.1 \pm 130.9 \\
{[2-780]}\end{array}$ & $\begin{array}{l}103.8 \pm 160.5 \\
{[2-780]}\end{array}$ \\
\hline $\begin{array}{l}\text { Age of onset of first depressive } \\
\text { episode (years) [range] }\end{array}$ & $\begin{array}{l}45.8 \pm 17.0 \\
{[16-89]}\end{array}$ & $\begin{array}{l}45.1 \pm 16.6 \\
{[16-76]}\end{array}$ \\
\hline$\%$ Treatment resistant $^{\mathrm{a}}$ & $70.6(n=36)$ & $68.8(n=22)$ \\
\hline CIRS-G total score & $6.9 \pm 4.2$ & $7.0 \pm 2.9$ \\
\hline BSI - Anxiety score & $1.7 \pm 1.0$ & $1.6 \pm 0.9$ \\
\hline \multicolumn{3}{|l|}{$\%$ Concurrent medication use } \\
\hline Antidepressant & $51.0(n=26)$ & $53.1(n=17)$ \\
\hline Benzodiazepine & $25.5(n=13)$ & $25.0(n=8)$ \\
\hline Both & $15.7(n=8)$ & $15.6(n=5)$ \\
\hline$\%$ Comorbid anxiety disorder & $35.3(n=18)$ & $28.1(n=9)$ \\
\hline \multicolumn{3}{|l|}{ Neurocognitive data } \\
\hline $\begin{array}{l}\text { CWIT condition } 3 \text { (inhibition) } \\
\text { weighted combined scaled score }\end{array}$ & $10.4 \pm 3.0$ & $10.3 \pm 2.9$ \\
\hline $\begin{array}{l}\text { CWIT condition } 1 \text { (color naming) } \\
\text { scaled score }\end{array}$ & $9.6 \pm 3.2$ & $9.6 \pm 2.3$ \\
\hline $\begin{array}{l}\text { TMT condition } 4 \text { vs. condition } \\
5 \text { scaled score }\end{array}$ & $8.7 \pm 3.0$ & $8.6 \pm 3.3$ \\
\hline $\begin{array}{l}\text { RBANS immediate memory } \\
\text { index score }\end{array}$ & $100.3 \pm 16.3$ & $101.0 \pm 15.9$ \\
\hline $\begin{array}{l}\text { RBANS delayed memory } \\
\text { index score }\end{array}$ & $100.0 \pm 13.2$ & $101.0 \pm 12.3$ \\
\hline RBANS modified total score & $98.6 \pm 14.5$ & $99.1 \pm 12.1$ \\
\hline Mini Mental State Examination & $28.7 \pm 1.2$ & $28.7 \pm 1.2$ \\
\hline
\end{tabular}

PAS paired associative stimulation, MADRS Montgomery-Asberg Depression Rating Scale, CIRS-G Cumulative Illness Rating Scale for Geriatrics, BSI Brief Symptom Inventory, CWIT Color-Word Interference Test, TMT Trail Making Test, RBANS Repeatable Battery for the Assessment of Neuropsychological Status

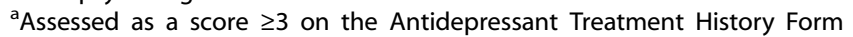
(non-response to a previous adequate antidepressant trial)

contraction). The electromyography signal was averaged across trials and the silent period duration was calculated from the start of the MEP to the return of electromyography activity by an experienced rater masked to the patient number.

PAS-induced changes in cortical excitability were measured as changes in the MEP amplitude in response to single pulses of TMS. Twenty TMS pulses were delivered at $0.1 \mathrm{~Hz}$ prior to and at four time points following the PAS intervention (i.e., pre-PAS, and immediately, $15 \mathrm{~min}, 30 \mathrm{~min}$, and $60 \mathrm{~min}$ post-PAS). The PAS intervention consisted of 180 trials at $0.1 \mathrm{~Hz}$ in which stimulation of the right median nerve was paired with a TMS test pulse to the left motor cortex $25 \mathrm{~ms}$ later [29]. Peak-to-peak MEP amplitudes were averaged at each time point. Average and maximum cortical plasticity were calculated as ratios of the average or maximum post-PAS MEP amplitudes/pre-PAS MEP amplitudes. 
Attention to the target hand during PAS is important for the induction of LTP-like cortical plasticity [44]. Patients were asked to focus their attention on the target hand during the PAS intervention and count the number of peripheral nerve stimulations. Their count was recorded randomly approximately 8 times during the PAS intervention. A measure of inattention was calculated over $i$ time points as:

$\sum \mid\left(\right.$ realCount $_{i}-$ realCount $\left._{i-1}\right)-\left(\right.$ guessCount $_{i}-$ guessCount $\left._{i-1}\right) \mid$.

A higher score reflects greater inattention, as a function of greater count errors throughout the PAS procedure. Count errors at each time point contribute to the inattention score, but are not carried forward in the calculation of later errors.

\section{Cognitive measures}

Two executive functions were studied here: cognitive flexibility (set shifting) and cognitive inhibition, as assessed with the Delis-Kaplan Executive Function System (D-KEFS) Trail Making Test and Color-Word Interference Test, respectively [45]. The Trail Making Test condition 4 (number-letter switching; similar to the Trails B Test) is a visual-motor sequencing task, in which patients are instructed to draw a line connecting numbers and letters in an alternating sequence (e.g., 1-a-2-b). In condition 5 (motor speed) of the Trail Making Test, the patient draws a line over a dotted line as quickly as possible. We report an age-corrected scaled score of condition 4 vs. condition 5 to account for motor speed (D-KEFS scaled scores are based on a mean of 10, standard deviation [SD] of 3). The D-KEFS Color-Word Interference Test condition 3 is based on the Stroop test; patients are presented with the names of colors printed in incongruently colored ink (e.g., "red" printed in blue ink), and the patient is required to name the color of the ink as quickly as possible, while inhibiting the prepotent tendency to read the word. Raw scores were converted to age-corrected scaled scores, and the final scaled score used here combines task completion time with number of errors committed (reported with permission from Pearson Inc.). The D-KEFS Trail Making Test and Color-Word Interference Test consisted of other conditions and scores, which were beyond the current scope of this study.

As control measures, immediate and delayed recall memory were assessed using the Repeatable Battery for the Assessment of Neuropsychological Status (RBANS) immediate memory and modified delayed memory index scores $[46,47]$. The immediate memory index assessed the immediate recall of a 10-item word list and 12-item short story (List Learning and Story Memory subtests), and the delayed memory index assessed the recall of the word list, story and a figure from the Figure Copy subtest $\sim 15$ min later (List Recall, List Recognition, Story Recall, and Figure Recall subtests). The RBANS included other subtests and domainspecific index scores (e.g., language, attention, and visuospatial/ constructional), but these measures were not the focus of the current study. To further test the specificity of any relationships with executive functioning, processing speed was also assessed using the D-KEFS Color-Word Interference Test condition 1 (color naming) scaled scores. Additionally, overall cognitive functioning was measured with the Mini Mental State Examination and the RBANS modified total score.

\section{Statistical analysis}

The assumption of normality was assessed for each measure using histograms, boxplots and Q-Q plots, as well as the D'Agostino-Pearson normality test. All cognitive measures (cognitive inhibition, set shifting, immediate memory, delayed memory, and processing speed) were normally distributed. Of the TMS measures, cortical silent period duration was normally distributed, whereas average and max post-PAS plasticity, PAS inattention score, resting motor threshold, SICI and ICF were found to be non-normally distributed, and a log transformation was applied to attain a normal distribution.

Six TMS measures (average and max PAS-induced plasticity, $\mathrm{SICl}$, ICF, cortical silent period, and resting motor threshold) were correlated with two measures of executive functioning (cognitive inhibition and set shifting), two measures of memory as a control (immediate and delayed recall memory), and a measure of processing speed as a control (color naming). After Bonferroni correction, the significance level was set at $p<0.002(0.05 / 30)$. Pearson's correlation coefficients $\left(r_{p}\right)$ were calculated. When assessing associations with cortical plasticity, partial correlations were performed, controlling for attention during the PAS procedure.

Sensitivity analyses were also performed to assess the influence of the following demographic and clinical variables: handedness, benzodiazepine use, treatment resistance, and comorbid anxiety disorder diagnosis. Any influence of the timing of cognitive and TMS testing was also examined (those patients who underwent cognitive and TMS testing on the same day vs. different days), since PAS has been found to influence response inhibition [48].

\section{RESULTS}

\section{Participants}

Cognition and cortical inhibition/excitation were assessed in 51 patients with LLD $(30 \mathrm{~F} / 21 \mathrm{M}, 66.6 \pm 5.9$ years of age). The PAS paradigm was initiated partway through the study, thus cortical plasticity was also assessed in 32 of these patients (17F/15M, 67.2 \pm 5.2 years of age). One patient did not complete the D-KEFS Trail Making Test, and cortical silent period duration is missing for one patient. The LLD patients investigated here showed normal overall cognitive functioning, as indicated by Mini Mental State Examination scores and RBANS total scaled scores. Patient demographic, clinical, and neurocognitive data are presented in Table 1.

Associations between neurophysiology and cognition Moderately strong partial correlations were identified between cognitive inhibition and both average PAS-induced plasticity $\left(r_{p}=-0.56, p<0.001\right.$, see Fig. 2$)$ and max PAS-induced plasticity $\left(r_{p}=-0.55, \quad p=0.001\right)$; both were significant after Bonferroni correction. By contrast, although set shifting showed the same direction of correlation with cortical plasticity, no significant correlations were observed between average or max plasticity and set shifting ( $r_{p}=-0.18, p=0.33 ; r_{p}=-0.18, p=0.35$, respectively). Similarly, no significant correlations were found between average or max plasticity and immediate memory $\left(r_{p}=-0.11\right.$, $p=0.57 ; \quad r_{p}=-0.17, p=0.35$, respectively), delayed memory $\left(r_{p}=-0.15, p=0.42 ; r_{p}=-0.15, p=0.43\right.$, respectively), or processing speed $\left(r_{p}=-0.35, p=0.053 ; r_{p}=-0.30, p=0.11\right.$, respectively). TMS measures of cortical inhibition ( $\mathrm{SICl}$ and cortical silent period) and excitation (ICF and resting motor threshold) were not significantly correlated with cognitive inhibition, set shifting, immediate memory, delayed memory, or processing speed (all $p>0.05)$.

The mean \pm SD TMS test pulse intensity was $64.2 \pm 15.8 \%$ of the maximum stimulator output. The mean MEP amplitude in response to the test pulse alone was $0.99 \pm 0.54 \mathrm{mV}$. Of note, age did not show any correlations with cognitive or TMS measures, other than a weak correlation with cognitive inhibition $\left(r_{p}=-0.30, p=0.036\right.$, uncorrected). However, a partial correlation with both inattention during PAS and age as covariates still yielded a significant correlation between cognitive inhibition and cortical plasticity (average plasticity: $r_{p}=-0.54, p=0.002$ ). MADRS scores also did not show any significant correlations between cognitive or TMS measures (all $p>0.2$ ).

To further explore the link between cognitive inhibition and cortical plasticity, patients were divided into those who did and did not show significant PAS-induced potentiation of cortical 


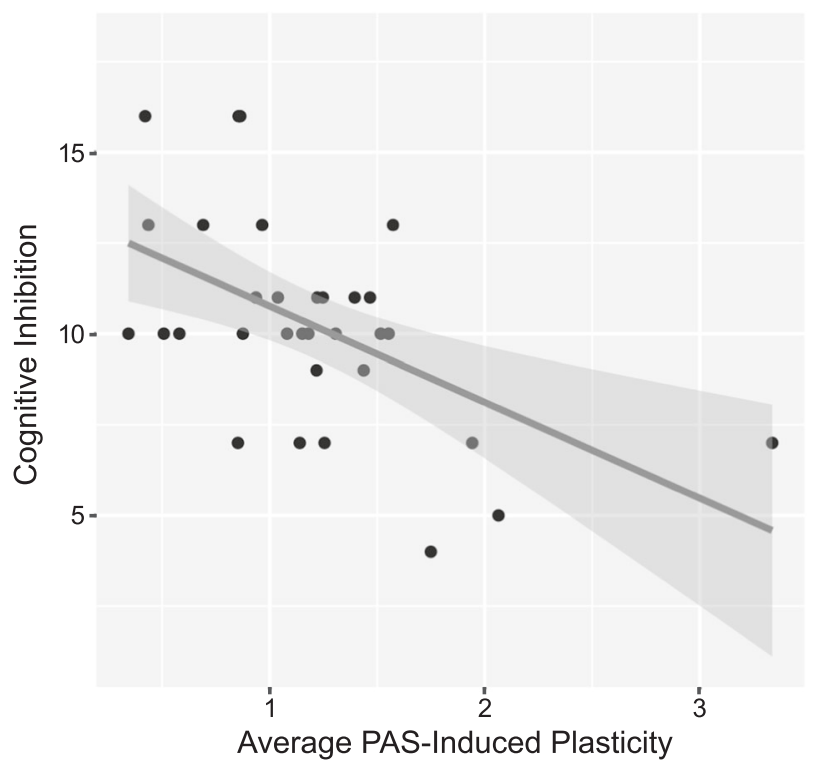

Fig. 2 Scatterplot of cognitive inhibition scaled scores, assessed using the Delis-Kaplan Executive Function System (D-KEFS) ColorWord Interference ("Stroop") Test, and average long-term potentiation (LTP)-like cortical plasticity induced by paired associative stimulation (PAS). Data points represent individual patients. A higher cognitive inhibition score reflects a greater ability to inhibit prepotent responses, and a higher PAS-induced plasticity ratio reflects greater potentiation of cortical output following the PAS paradigm. For visualization purposes, the gray line represents a least-squares linear fit with standard error confidence intervals

excitability ( $n=17$ and $n=15$, respectively). For each post-PAS time point, a paired $t$ test compared the baseline MEP amplitudes of 20 pre-PAS trials to the MEP amplitudes of 20 post-PAS trials. Significant PAS-induced plasticity was defined as a significant $(p<$ 0.05 ) increase in MEP amplitudes from pre- to post-PAS at one or more post-PAS time points. A significant difference in cognitive inhibition was observed between patients who did and did not show significant PAS-induced cortical plasticity (two-tailed independent $t$ test, $t_{30}=3.49, p=0.002$ ); patients with significant PASinduced changes in cortical excitability showed lower cognitive inhibition $\left(d_{\text {Cohen }}=1.24\right)$. By contrast, no significant differences in set shifting, memory, or processing speed were observed between groups $(p>0.3)$.

\section{Sensitivity analyses}

The sensitivity analyses indicated that the association between cognitive inhibition and cortical plasticity was robust in the presence/absence of a number of demographic, clinical and methodological variables. A moderately strong partial correlation between cognitive inhibition and cortical plasticity remained when the analyses included only: right-handed participants $\left(n=29\right.$; average plasticity: $r_{p}=-0.57, p=0.001$; max plasticity: $\left.r_{p}=-0.55, p=0.002\right)$, patients benzodiazepine free at the time of the study $\left(n=23\right.$; average plasticity: $r_{p}=$ $-0.59, p=0.004$; max plasticity: $\left.r_{p}=-0.63, p=0.002\right)$, patients without a comorbid anxiety disorder $(n=23$; average plasticity: $r_{p}=-0.58, p=0.005 ; \max$ plasticity: $\left.r_{p}=-.53, p=0.012\right)$, and treatment-resistant patients $\left(n=23\right.$; average plasticity: $r_{p}$ $=-0.61, p=0.003$; max plasticity: $r_{p}=-.61, p=0.002$ ). Additionally, patients who underwent TMS and cognitive testing on the same day $\left(n=11\right.$; average plasticity: $r_{p}=-0.70, p=0.025$; max plasticity: $\left.r_{p}=-.66, p=0.036\right)$, and those who underwent TMS and cognitive testing on different days $(n=21$; average plasticity: $r_{p}=-0.44, p=0.053 ; \max$ plasticity: $r_{p}=-.46$, $p=0.043$ ), showed similar negative correlations between cognitive inhibition and LTP-like cortical plasticity. Lastly, the association between cognitive inhibition and cortical plasticity remained significant when a PAS outlier (highest PAS measurement, Fig. 2) was removed ( $n=31$; average plasticity: $r_{p}=-0.57$, $p=0.001$; max plasticity: $r_{p}=-0.53, p=0.003$ ).

\section{DISCUSSION}

Using TMS paradigms to investigate the neural correlates of executive functions in LLD offers an opportunity to better understand the pathophysiology of LLD by breaking LLD down into its tractable dimensions. Our findings demonstrate an association between lower cognitive inhibition, that is, a reduced ability to inhibit prepotent responses, and higher LTP-like cortical plasticity in LLD. Consistent with our hypotheses, this relationship was specific to executive functioning, and was not observed for recall memory or processing speed. Contrary to our hypotheses, cognitive flexibility showed the same direction of association with cortical plasticity, but the association was not significant. Similarly, other neurophysiological processes, namely baseline cortical inhibition and excitation, were not found to be associated with cognitive inhibition, cognitive flexibility, episodic memory, or processing speed.

The observed relationship between lower cognitive inhibition and higher PAS-induced cortical potentiation suggests that LLD patients with healthy levels of cognitive inhibition show more stable levels of cortical excitability following repeated firing of cortical circuits. Conversely, the direction of this association suggests that elevated potentiation of cortical excitability upon repeated synaptic firing may be involved in the inhibitory control deficits seen in some patients with $\operatorname{LLD}[2-4,8,12]$. Consistent with our findings, a relationship between poorer cognitive inhibition and hyperactivity in frontal cortical regions has been observed in younger patients with depression, and not healthy controls [27]. Similarly, Daselaar et al. [49] found an association between poorer executive functioning and greater cortical firing during an executive functioning task in older adults. Considering our findings within the broader literature, it is possible that in some LLD patients, repeated firing of maladaptive cortical circuits allows prepotent, inappropriate responses to become overly facilitated, and thus more difficult to inhibit. Alternatively, given that the prolonged elevation of NMDA receptor activation in the brain can produce excitotoxicity, causing neuronal injury [50], it is also possible that the facilitated induction of elevated cortical excitability could promote excitotoxicity [51] within cortical circuits important for executive functioning. We should note, however, that there were too few patients in the current sample showing below-average cognitive inhibition scores to draw conclusions specific to LLD patients with executive dysfunction. The inverse relationship observed here between cognitive inhibition and cortical plasticity highlights the importance of efficient cortical plasticity, in which stable levels of total excitation are maintained, for optimal cognitive functioning [52].

Our observation that cognitive inhibition, a core executive function, is related to cortical plasticity is also in line with evidence that novel plasticity-based interventions can influence executive functioning in LLD patients. For example, intensive cognitive interventions, such as neuroplasticity-based computerized cognitive remediation for geriatric depression, influence functional plasticity in the aging brain [53,54], and improve executive functions, including cognitive inhibition, in LLD patients [55]. Similarly, repetitive TMS treatment has been shown to both induce cortical plasticity [56] and improve executive functions such as cognitive inhibition in non-elderly patients with depression $[57,58]$. In contrast, traditional antidepressant treatments do not influence executive functioning in LLD [13]. The preliminary findings from plasticity-based treatments raise the possibility of a 
1664

close mechanistic association between cortical plasticity and cognitive inhibition in LLD.

Both cognitive inhibition and cortical plasticity showed considerable variability in the LLD patients we studied (Fig. 2). Similarly, in previous studies approximately half of LLD patients exhibited normal cognitive functioning, including in the executive domain $[6,9]$. This variability might explain why some studies do not find a relationship between LLD and abnormal LTP-like cortical plasticity [24] or deficits in cognitive inhibition [59]. Within this variability, it is possible that there is a distinct subpopulation of LLD patients who demonstrate lower cognitive inhibition and elevated LTP-like cortical plasticity. Although speculative, the possibility that neurobiological/ cognitive subtypes of LLD exist is in line with evidence that LLD patients with and without executive dysfunction show different clinical profiles [60].

Surprisingly, the relationship with cortical plasticity was specific to cognitive inhibition, and not cognitive flexilibity. While both cognitive processes are components of executive functioning, cognitive inhibition and flexibility have been shown to have both shared and distinct neural substrates [61], and may accordingly play distinct roles in the pathophysiology and prognosis of LLD. For example, in a previous study of LLD patients, cognitive flexibility, and not cognitive inhibition, was found to be predictive of clinical response to aripiprazole augmentation [62]. It is plausible that cortical plasticity is involved in inhibitory control, but not the flexible switching between tasks, which may involve other neurobiological mechanisms. Nevertheless, our findings require replication due to the crosssectional design of the current study.

Additional limitations of the current study should be considered. (i) First, the current report cannot comment on the specificity of the observed relationship to LLD; an investigation of the relationship between cognitive inhibition and cortical plasticity in healthy older adults and patients with other psychiatric disorders is needed. (ii) Second, we assessed neurophysiological measures in the motor cortex, whereas subregions of the prefrontal cortex are most commonly associated with LLD pathology and executive functions. Nevertheless, the neocortex shows similar cellular organization and patterns of connections across cortical areas [63]. Abnormal motor cortical plasticity has also been reported in patients with depression using TMS [19]. Future LLD studies could utilize TMS combined with EEG to assess the relationship between executive functions and cortical plasticity, inhibition, and excitation in the prefrontal cortex, as was done in a recent PAS study of the relationship between working memory and dorsolateral prefrontal cortex plasticity in Alzheimer's disease [64]. (iii) Third, the number of patients in this sample with cognitive inhibition deficits is insufficient to draw conclusions specific to LLD patients with executive dysfunction. (iv) Fourth, due to the cross-sectional nature of this study, the relationship of the current measures to treatment outcome was not addressed here, and should be assessed in future studies. (v) Finally, given the high proportion of medicated and treatmentresistant patients studied here, we cannot infer whether our observed association applies differently to medication-free and non-treatment-resistant LLD patients.

To our knowledge, this is the first study to use TMS to investigate the association between specific endophenotypes of LLD and cortical physiology. It is also the first report of a relationship between cognitive inhibitory control (top-down executive functioning) and cortical plasticity in LLD. The direction of association observed here suggests that in patients with reduced cognitive inhibition, repeated activation of the same cortical regions may temporarily promote hyper-excitable output of these regions. By uncovering the cortical mechanisms specific to executive functioning in LLD, the present work may help to inform the development of treatments for LLD that address this key cognitive dimension.

\section{FUNDING AND DISCLOSURES}

This study was funded in part by a Brain and Behavior Research Foundation New Investigator Award (DMB), the Canadian Institutes of Health Research (CIHR; MOP-123455), and the National Institutes of Health (NIH; R34MH101365). We would also like to acknowledge the Temerty Center and the Canada Foundation for Innovation for providing TMS equipment. TKR has received research support from Brain Canada, Brain and Behavior Research Foundation, BrightFocus Foundation, Canada Foundation for Innovation, Canada Research Chair, CIHR, Center for Aging and Brain Health Innovation, $\mathrm{NIH}$, Ontario Ministry of Health and Long-Term Care, Ontario Ministry of Research and Innovation, and the Weston Brain Institute. JFK received medication supplies from Indivior to support this investigator-initiated trial. He has also received medication supplies from Pfizer for investigator-initiated work. He receives research funding from the $\mathrm{NIH}$ and Patient-Centered Outcomes Research Institute (PCORI). CFR has received research support from the NIH, PCORI, the Center for Medicare and Medicaid Services, the American Foundation for Suicide Prevention, the Brain and Behavior Research Foundation, and the Commonwealth of Pennsylvania. Bristol Meyers Squib and Pfizer have provided pharmaceutical supplies for his NIH sponsored research. EJL reports research funding (current/past) from Janssen, Alkermes, Acadia, Takeda, Lundbeck, Barnes Jewish Foundation, PCORI, and Taylor Family Institute for Innovative Psychiatric Research. In the past 5 years, ZJD has received research and equipment in-kind support for an investigator-initiated study through Brainsway Inc. and Magventure Inc. His work was supported by the Ontario Mental Health Foundation (OMHF), CIHR, the NIMH, and the Temerty Family and Grant Family and through the CAMH Foundation and the Campbell Institute. BHM currently receives research funding from Brain Canada, the CAMH Foundation, PCORI, and the NIH. During the past 5 years, he also received research funding from CIHR, and support in kind from Capital Solution Design LLC (software used in a study funded by CAMH Foundation), HAPPYneuron (software used in a study funded by Brain Canada), Bristol-Myers Squibb (medications for a $\mathrm{NIH}$-funded clinical trial), Eli-Lilly (medications for a $\mathrm{NIH}$-funded clinical trial), and Pfizer (medications for a $\mathrm{NIH}$ funded clinical trial). He directly owns stocks of General Electric $(<\$ 5000)$. DMB has received research support from $\mathrm{CIHR}, \mathrm{NIH}$, Brain Canada. and the Temerty Family through the CAMH Foundation and the Campbell Research Institute. He receives research support and in-kind equipment support for an investigator-initiated study from Brainsway Ltd. and he is the site principal investigator for three sponsor-initiated studies for Brainsway Ltd. He also receives in-kind equipment support from Magventure for an investigator-initiated study. He receives medication supplies for an investigator-initiated trial from Indivior. The other authors declare no competing interests.

\section{ADDITIONAL INFORMATION}

Publisher's note: Springer Nature remains neutral with regard to jurisdictional claims in published maps and institutional affiliations.

\section{REFERENCES}

1. Diamond A. Executive functions. Annu Rev Psychol. 2013;64:135-68.

2. Lesser IM, Boone KB, Mehringer CM, Wohl MA. Cognition and white matter hyperintensities in older depressed patients. Am J Psychiatry. 1996;153:1280-87.

3. Kindermann SS, Kalayam B, Brown GG, Burdick KE, Alexopoulos GS. Executive functions and P300 latency in elderly depressed patients and control subjects. Am J Geriatr Psychiatry. 2000;8:57-65.

4. Lockwood KA, Alexopoulos GS, van Gorp WG. Executive dysfunction in geriatric depression. Am J Psychiatry. 2002;159:1119-26.

5. Dybedal GS, Tanum L, Sundet K, Gaarden TL, Bjølseth TM. Neuropsychological functioning in late-life depression. Front Psychol. 2013;4:381. 
6. Koenig AM, DeLozier IJ, Zmuda MD, Marron MM, Begley AE, Anderson SJ, et al. Neuropsychological functioning in the acute and remitted states of late-life depression. J Alzheimers Dis. 2015;45:175-85.

7. Katz R, De Sanctis P, Mahoney JR, Sehatpour P, Murphy CF, Gomez-Ramirez M, et al. Cognitive control in late-life depression: response inhibition deficits and dysfunction of the anterior cingulate cortex. Am J Geriatr Psychiatry. 2010;18:1017-25.

8. Klojčnik $M$, Kavcic V, Bakracevic Vukman K. Relationship of depression with executive functions and visuospatial memory in elderly. Int J Aging Hum Dev. 2017;85:490-503.

9. Butters MA, Whyte EM, Nebes RD, Begley AE, Dew MA, Mulsant BH, et al. The nature and determinants of neuropsychological functioning in late-life depression. Arch Gen Psychiatry. 2004;61:587-95.

10. Thomas AJ, Gallagher P, Robinson LJ, Porter RJ, Young AH, Ferrier IN, et al. A comparison of neurocognitive impairment in younger and older adults with major depression. Psychol Med. 2009;39:725-33.

11. Alexopoulos GS, Kiosses DN, Heo M, Murphy CF, Shanmugham B, Gunning-Dixon F. Executive dysfunction and the course of geriatric depression. Biol Psychiatry. 2005;58:204-10.

12. Richard-Devantoy S, Jollant F, Kefi Z, Turecki G, Olié JP, Annweiler C, et al. Deficit of cognitive inhibition in depressed elderly: neurocognitive marker of suicidal risk. J Affect Disord. 2012;140:193-9.

13. Nebes RD, Pollock BG, Houck PR, Butters MA, Mulsant BH, Zmuda MD, et al. Persistence of cognitive impairment in geriatric patients following antidepressant treatment: a randomized, double-blind clinical trial with nortriptyline and paroxetine. J Psychiatr Res. 2003;37:99-108.

14. Murphy CF, Gunning-Dixon FM, Hoptman MJ, Lim KO, Ardekani B, Shields JK, et al. White-matter integrity predicts stroop performance in patients with geriatric depression. Biol Psychiatry. 2007;61:1007-10.

15. Li W, Wang Y, Ward BD, Antuono PG, Li S-J, Goveas JS. Intrinsic inter-network brain dysfunction correlates with symptom dimensions in late-life depression. J Psychiatr Res. 2017;87:71-80.

16. Zhang B-W, Zhao L, Xu J. Electrophysiological activity underlying inhibitory control processes in late-life depression: a Go/Nogo study. Neurosci Lett. 2007;419:225-30.

17. Abbott LF, Nelson SB. Synaptic plasticity: taming the beast. Nat Neurosci. 2000;3:1178-83.

18. Isaacson J, Scanziani M. How inhibition shapes cortical activity. Neuron. 2011;72:231-43.

19. Player MJ, Taylor JL, Weickert CS, Alonzo A, Sachdev P, Martin D, et al. Neuroplasticity in depressed individuals compared with healthy controls. Neuropsychopharmacology. 2013;38:2101-8.

20. Bajbouj M, Lisanby SH, Lang UE, Danker-Hopfe H, Heuser I, Neu P. Evidence for impaired cortical inhibition in patients with unipolar major depression. Biol Psychiatry. 2006;59:395-400.

21. Tecchio F, Zappasodi F, Pasqualetti P, Gennaro LD, Pellicciari MC, Ercolani M, et al. Age dependence of primary motor cortex plasticity induced by paired associative stimulation. Clin Neurophysiol. 2008;119:675-82.

22. Cueva AS, Galhardoni R, Cury RG, Parravano DC, Correa G, Araujo H, et al. Normative data of cortical excitability measurements obtained by transcranial magnetic stimulation in healthy subjects. Clin Neurophysiol. 2016;46:43-51.

23. Lissemore Jl, Bhandari A, Mulsant BH, Lenze EJ, Reynolds CF, Karp JF, et al. Reduced GABAergic cortical inhibition in aging and depression. Neuropsychopharmacology. 2018;43:2277-84.

24. Bhandari A, Lissemore Jl, Rajji TK, Mulsant BH, Cash RFH, Noda Y, et al. Assessment of neuroplasticity in late-life depression with transcranial magnetic stimulation. J Psychiatr Res. 2018;105:63-70.

25. Alexopoulos GS, Schultz SK, Lebowitz BD. Late-life depression: a model for medical classification. Biol Psychiatry. 2005;58:283-9.

26. Miller EK, Cohen JD. An integrative theory of prefrontal cortex function. Ann Rev Neurosci. 2001;24:167-202.

27. Wagner G, Sinsel E, Sobanski T, Köhler S, Marinou V, Mentzel H-J, et al. Cortical inefficiency in patients with unipolar depression: an event-related fMRI study with the Stroop task. Biol Psychiatry. 2006;59:958-65.

28. Markram H, Lübke J, Frotscher M, Sakmann B. Regulation of synaptic efficacy by coincidence of postsynaptic APs and EPSPs. Science. 1997;275:213-15.

29. Stefan K, Kunesch E, Cohen LG, Benecke R, Classen J. Induction of plasticity in the human motor cortex by paired associative stimulation. Brain. 2000; 123:572-84.

30. Stefan K, Kunesch E, Benecke R, Cohen LG, Classen J. Mechanisms of enhancement of human motor cortex excitability induced by interventional paired associative stimulation. J Physiol. 2002;543:699-708.

31. Kujirai T, Caramia MD, Rothwell JC, Day BL, Thompson PD, Ferbert A, et al. Corticocortical inhibition in human motor cortex. J Physiol. 1993;471:501-19.
32. Paulus W, Classen J, Cohen LG, Large CH, Di Lazzaro V, Nitsche M, et al. State of the art: pharmacologic effects on cortical excitability measures tested by transcranial magnetic stimulation. Brain Stimul. 2008;1:151-63.

33. Cantello R, Gianelli M, Civardi C, Mutani R. Magnetic brain stimulation: the silent period after the motor evoked potential. Neurology. 1992;42:1951-9.

34. Radhu N, de Jesus DR, Ravindran LN, Zanjani A, Fitzgerald PB, Daskalakis ZJ. A meta-analysis of cortical inhibition and excitability using transcranial magnetic stimulation in psychiatric disorders. Clin Neurophysiol. 2013;124:1309-20.

35. Lenze EJ, Mulsant BH, Blumberger DM, Karp JF, Newcomer JW, Anderson SJ, et al. Efficacy, safety, and tolerability of augmentation pharmacotherapy with aripiprazole for treatment-resistant depression in late life: a randomised, doubleblind, placebo-controlled trial. Lancet. 2015;386:2404-12.

36. Joel I, Begley AE, Mulsant BH, Lenze EJ, Mazumdar S, Dew MA, et al. Dynamic prediction of treatment response in late-life depression. Am J Geriatr Psychiatry. 2014;22:167-76.

37. First MB, Spitzer RL, Gibbon M, Williams JB. Structured clinical interview for DSM-IV axis I disorders. New York State Psychiatric Institute, Biometrics Research: New York; 1995.

38. Montgomery SA, Asberg M. A new depression scale designed to be sensitive to change. Br J Psychiatry. 1979;134:382-9.

39. Folstein M, Folstein S, McHugh P. "Mini-mental state": a practical method for grading the cognitive state of patients for the clinician. J Psychiatr Res. 1975;12:189-98.

40. Sackeim HA. The definition and meaning of treatment-resistant depression. J Clin Psychiatry. 2001;62:10-7.

41. Miller MD, Paradis CF, Houck PR, Mazumdar S, Stack JA, Rifai AH, et al. Rating chronic medical illness burden in geropsychiatric practice and research: application of the Cumulative Illness Rating Scale. Psychiatry Res. 1992;41:237-48.

42. Derogatis LR, Melisaratos N. The brief symptom inventory: an introductory report. Psychol Med. 1983;13:595-605.

43. Chipchase L, Schabrun S, Cohen L, Hodges P, Ridding M, Rothwell J, et al. A checklist for assessing the methodological quality of studies using transcranial magnetic stimulation to study the motor system: $\mathrm{n}$ international consensus study. Clin Neurophysiol. 2012;123:1698-704.

44. Stefan K, Wycislo M, Classen J. Modulation of associative human motor cortical plasticity by attention. J Neurophysiol. 2004;92:66-72.

45. Delis DC, Kaplan E, Kramer JH. Delis-Kaplan executive function system: examiner's manual. San Antonio, TX: Psychological Corporation; 2001.

46. Randolph C. The Repeatable Battery for the Assessment of Neuropsychological Status. San Antonio: The Psychological Corporation; 1998.

47. Duff K, Patton D, Schoenberg MR, Mold J, Scott JG, Adams RL. Age-and education-corrected independent normative data for the RBANS in a community dwelling elderly sample. Clin Neuropsychol. 2003;17:351-66.

48. Kohl S, Hannah R, Rocchi L, Nord CL, Rothwell J, Voon V. Cortical paired associative stimulation influences response inhibition: cortico-cortical and corticosubcortical networks. Biol Psychiatry. 2019;85:355-63.

49. Daselaar SM, lyengar V, Davis SW, Eklund K, Hayes SM, Cabeza RE. Less wiring more firing: low-performing older adults compensate for impaired white matter with greater neural activity. Cereb Cortex. 2015;25:983-90.

50. Choi DW. Glutamate neurotoxicity and diseases of the nervous system. Neuron. 1988;1:623-34.

51. McEachern JC, Shaw CA. The plasticity-pathology continuum: defining a role for the LTP phenomenon. J Neurosci Res. 1999;58:42-61.

52. Pascual-Leone A, Freitas C, Oberman L, Horvath JC, Halko M, Eldaief M, et al. Characterizing brain cortical plasticity and network dynamics across the agespan in health and disease with TMS-EEG and TMS-fMRI. Brain Topogr. 2011;24:302-15.

53. Erickson KI, Colcombe SJ, Wadhwa R, Bherer L, Peterson MS, Scalf PE, et al. Training-induced plasticity in older adults: effects of training on hemispheric asymmetry. Neurobiol Aging. 2007;28:272-83.

54. Mozolic J, Hayaska S, Laurienti P. A cognitive training intervention increases resting cerebral blood flow in healthy older adults. Front Hum Neurosci. 2010;4:1-10.

55. Morimoto SS, Wexler BE, Liu J, Hu W, Seirup J, Alexopoulos GS. Neuroplasticitybased computerized cognitive remediation for treatment-resistant geriatric depression. Nat Commun. 2014;5:4579.

56. Fitzgerald PB, Fountain S, Daskalakis ZJ. A comprehensive review of the effects of rTMS on motor cortical excitability and inhibition. Clin Neurophysiol. 2006;117:2584-96.

57. Blumberger DM. Can repetitive transcranial magnetic stimulation enhance cognitive control in late-life depression? Am J Geriatr Psychiatry. 2018;26:347-49.

58. Ilieva IP, Alexopoulos GS, Dubin MJ, Morimoto SS, Victoria LW, Gunning FM. Age-related repetitive transcranial magnetic stimulation effects on executive function in depression: a systematic review. Am J Geriatr Psychiatry. 2018;26:334-46. 
59. Alexopoulos GS, Manning K, Kanellopoulos D, McGovern A, Seirup JK, Banerjee S, et al. Cognitive control, reward-related decision making and outcomes of late-life depression treated with an antidepressant. Psychol Med. 2015;45:3111-20.

60. Alexopoulos GS, Kiosses DN, Klimstra S, Kalayam B, Bruce ML. Clinical presentation of the "depression-executive dysfunction syndrome" of late life. Am J Geriatr Psychiatry. 2002;10:98-106.

61. Hedden T, Gabrieli JDE. Shared and selective neural correlates of inhibition, facilitation, and shifting processes during executive control. Neuroimage. 2010;51:421-31.
62. Kaneriya SH, Robbins-Welty GA, Smagula SF, et al. Predictors and moderators of remission with aripiprazole augmentation in treatment-resistant late-life depression: an analysis of the irl-grey randomized clinical trial. JAMA Psychiatry. 2016;73:329-36.

63. Harris KD, Shepherd GMG. The neocortical circuit: themes and variations. Nat Neurosci. 2015;18:170-81.

64. Kumar S, Zomorrodi R, Ghazala Z, Goodman MS, Blumberger DM, Cheam A, et al. Extent of dorsolateral prefrontal cortex plasticity and its association with working memory in patients with Alzheimer disease. JAMA Psychiatry. 2017;74:1266-74. 